Vie
- d'inciter les têtes de réseaux associatifs (associations de jeunesse et d'éducation populaire) à s'emparer de ce sujet, à l'intégrer à leurs projets et à le relayer auprès des associations adhérentes, et d'inciter ces associations à monter des projets locaux et à diffuser l'information sur les changements climatiques;

- de déclarer une année de la lutte contre l'effet de serre, sous l'égide de l'ONU;

- de déclarer une journée nationale de la lutte contre l'effet de serre avec actions diverses (journée sans voiture, échanges d'expériences entre pays etc.) ;

- de créer une plaquette qui traduise en termes d'émissions de $\mathrm{CO}_{2}$ tous les actes de la vie courante, et qui permette d'évaluer les gains obtenus grâce aux changements de comportements. La distribution de cette plaquette doit être précédée d'une campagne d'information nationale ;

- de prendre des mesures nécessaires pour que les acquis ne soient pas remis en cause par le changement de majorité politique :

- de sensibiliser les enfants en milieu scolaire dès leur plus jeune âge : mise en place de projets au sein des écoles ;
- de généraliser ces enseignements à toutes les classes d'âges par des méthodes appropriées.

\section{Conclusion}

Les instances démocratiques traditionnelles ont montré leurs limites. La conférence citoyenne nous a permis d'explorer une nouvelle voie : la voie de la démocratie directe.

Le citoyen n'est pas un intermittent de la vie politique, mais l'acteur à part entière de son devenir. De même que les changements climatiques ne connaissent pas de frontières, la citoyenneté peut être le vecteur d'une solidarité universelle, seul comportement rationnel et responsable face à un choix crucial :

- soit nous continuons dans la voie actuelle d'une croissance économique forte et nous sacrifions les générations futures,

- soit nous modifions radicalement nos comportements et nous garantissons un développement durable de notre planète.

\title{
Les sciences de la conception. Enjeu scientifique du XXIe siècle
}

\section{Une conférence internationale en hommage à Herbert Simon}

\author{
MICHEL FILIPPI
}

\begin{abstract}
MICHEL FILIPPI Herbert Simon (1916-2001) affirma, après John
Philosophe Dewey et d'autres, que la pensée humaine construit École centrale de Lyon et alors connaît, connaît et alors construit. II testa ce Laboratoire ICTT qui n'était qu'hypothèse en explorant la pragmatique 36, avenue Guy-de- humaine dans des domaines comme le management Collongue-BP 163 (ou, plus généralement, la systémique), les sciences 69131 Ecully cedex de l'information, la « computique " (terme emprunté Michel.Filippi@ec-lyon.fr à J.L. Le Moigne), l'économie et la psychologie. Il en déduisit que rien de ce que fait l'Homme n'est de nature, que tout ce qu'il produit est artificiel. II démontra alors qu'une science de l'artificiel était possible, une science des modes de construction utilisés par I'Homme. II a montré que cette science était une science des systèmes complexes, et imposé l'idée que les systèmes de traitement de l'information sont une classe centrale de systèmes dans le monde.

En février 1984, s'était tenu à la Grande Motte, près

${ }^{1}$ Les actes de ce colloque ont été publiés aux PUL en 1986, mais le livre est épuisé (ISBN : 72970287 3).
\end{abstract}

qu'elle leur suscitait. Rappelons quelques-unes des réponses que fit le père des sciences de l'artificiel. Les programmes informatiques sont des théories que l'on peut tester; ils nous permettent de faire des hypothèses sur ce qui sous-tend, dans le cerveau, les processus de construction ; nous ne pouvons encore décider si ce sont les mêmes processus à l'œuvre dans l'homme qui sont responsables de l'apprentissage à court terme et du développement à long terme; la théorie du traitement de l'information n'est pas une théorie neuro-anatomique; le défi pour la théorie psychologique du traitement de l'information est de montrer que ce sont fondamentalement les mêmes types de processus qui interviennent, d'une part, pour trouver un itinéraire entre une situation de problème et sa solution et, d'autre part, pour trouver une voie menant d'une situation à la formulation d'un problème. Simon affirma de plus que l'attention humaine est une ressource bien plus rare que ne l'est l'information et que nous devons améliorer tout système de décision en y insérant des filtres intelligents. 
Attention limitée et raison limitée sont une même chose. En effet aucun homme ne peut choisir un mode d'action en computant toutes les informations disponibles dans le monde. $\mathrm{S}^{\prime}$ il le pouvait, il serait comme le démon de Laplace. Or, l'expérience montre que ce n'est pas le cas. Au lieu donc de théoriser un homme omnipotent, Simon par la théorie de la rationalité limitée, construisit une théorie du * comment vivre * dans un monde infini tout en ne disposant que de très modestes * moyens " de computations : ceux que nous fournissent notre environnement local. Cet environnement local est composé des contraintes externes, dont celles de nature (par exemple, le champ de blé est un objet artificiel, une construction (ou conception) des hommes, mais le blé pousse selon les lois de la nature), et de contraintes internes qui sont propres à l'objet construit (ou conçu) en computant les informations locales.

Remarquons alors que tout ce que produit l'Homme est objet quelle que soit la matière utilisée pour le faire.

Près de vingt ans plus tard, nous attendions à nouveau d'Herbert Simon des réponses à nos questions lors de la rencontre internationale, Les sciences de la conception, enjeu scientifique du $\mathrm{XXI}^{\mathrm{e}}$ siècle $^{2}$, tenue à l'INSA de Lyon (Villeurbanne, France). Non plus celles issues de notre lecture de l'œuvre, mais celles produites par son application. Hélas, la déception fut grande, car Herbert Simon mourut quelques mois avant qu'il ait pu nous répondre. Mais les questions étaient là : pour quelle raison Simon ne s'était-il pas préoccupé des problèmes mal définis? Pour quelle raison n'avait-il pas accordé de l'importance au mécanisme de représentation de problèmes ? Pourquoi ne s'être pas intéressé aux sauts * non déterministes * et aux obstacles que rencontrent la décomposition de problèmes ? ${ }^{3}$ Mais n'étaient-ce pas les questions que I'on voudrait poser au père fondateur avant qu'il ne disparaisse et nous laisse au travail, héritiers inquiets de l'œuvre à continuer, à trahir peut-être, à développer et à enrichir sûrement, à moins que, nous sentant trop faibles face à ce génie, nous n'ayons pas le courage de continuer. C'est pourquoi la question posée par Jacques Perrin, l'organisateur de cette rencontre, «quel programme de recherche pour les sciences de la conception? ", est fondamentale. Y répondre est le seul moyen de pouvoir continuer ce qui a été entrepris, quitte à trahir.

On ne peut en quelques lignes faire le tour de tous les travaux ${ }^{4}$ qui ont été présentés. La rencontre était vraiment internationale et presque tous les domaines explorés par les sciences de l'artificiel représentés. Seul manquait Edgar Morin invité par la Chine à la même époque pour exposer ses travaux et leurs conséquences. Mais il était présent par un texte, Le paradoxe de l'observateur-concepteur, qui tire les conséquences des sciences de l'artificiel pour l'Homme. Le concepteur, c'est-à-dire tout homme qui a la volonté de construire, se doit de concevoir la multiplicité des points de vue et le passage d'un point de vue à l'autre. Il doit disposer de concepts théoriques qui, au lieu de fermer et d'isoler les entités (physique, biologique, sociologique), lui permettent de circuler de manière productive : "Il doit concevoir en même temps I'individualité des êtres machinaux, les Machines des machines qui les englobent, et les complexes de machines interdépendantes ou polymachines qui les associent ". Ne pouvant plus être face à la nature (la connaissant sans être affecté par elle) ou en elle (entièrement déterminé par la nature), I'Homme devient un observateur-concepteur engagé « dans une problématique où sa vision du monde des machines met en cause à la fois sa vision du monde, la vision qu'il a de la société, la vision qui lui vient de la société ».

Cette vision de l'Homme était déjà présente dans l'œuvre de Giambattista Vico, philosophe italien du XVIII ${ }^{e}$ siècle, nous rappela J.-L. Le Moigne, I'un des introducteurs de l'œuvre de Simon en France, son traducteur et l'un de ses amis. Cette rationalité du monde s'était développée en Europe à partir du XVe siècle comme sciences du génie, ou ingenium, et fut abandonnée au profit des sciences analytiques dites cartésiennes. La domination des sciences analytiques a eu comme conséquence le discrédit jeté sur l'activité humaine comme moyen de connaissance, moyen de faire science. Le processus de création des objets en a été altéré à un point tel que l'on a cru que produire de nouveaux objets résulte de la seule application des lois de la nature. Les industriels ont commencé à abandonner ce modèle parce que les exigences du marché les y contraignent, pour le remplacer par les modèles de conception issus des sciences de l'artificiel. Mais les sciences de l'artificiel doivent s'intéresser à l'industrie, ne pas attendre qu'elle vienne à elles, parce qu'y sont produits les objets qui transforment le monde des hommes, qu'y apparaissent la modification des techniques qui font émerger les civilisations. Pour les mêmes raisons, elles doivent exercer leur influence dans le domaine de la médecine, du droit, de la gestion. Les sciences de l'artificiel doivent reconquérir le monde de la technè, celui de l'activité contingente, activité par laquelle l'Homme use de son habilité à saisir une réalité polymorphe grâce à son coup d'œil, et de cette saisie naît un objet, un acte, une relation qui existe alors, mais aurait pu ne pas exister ou être autre que ce qu'il (ou elle) est maintenant.

Le modèle de l'objet produit par l'activité contingente est le gouvernail, ensemble de pièces de bois liées ensemble. Aucune d'elles ne représente une théorie de la navigation; aucune d'elles, de par sa nature seule, ne contraint les autres à agir sur le navire; mais le coup d'œil du constructeur a saisi dans leur ensemble la possibilité pour les Hommes de mener leurs navires comme ils l'entendent. L'Homme utilise des morceaux pour construire et réutilise des parties de ce qu'il a construit afin de produire de la nouveauté. C'est un constat. On réutilise ce qui a des qualités particulières, testées par des usages anciens. Dans les domaines où la fiabilité des parties est abso- 2180 participants lument nécessaire, on cherchera plus facilement et 15 pays représentés. qu'ailleurs à utiliser du connu pour produire de la ${ }^{3}$ Willemien Visser de nouveauté. Les concepteurs de logiciels tentent de I'INRIA (projet EIFFEL). produire des pièces de programme, des morceaux ${ }^{4}$ Quarante communicaréutilisables car fiables. Cependant, ils sont affrontés tions disponibles sur $C D$

à la réalité changeante comme le furent les Grecs Rom. Le commander anciens : un logiciel n'est pas que le résultat de toutes ses parties. Ce serait une vision des sciences analytiques : le logiciel comme objet logiquement déterminé. On doit se demander si le "patterning ", le fait joelle.forest@insa-lyon.fr. Centre des Humanités, Insa, 20, bd Einstein 
74. Scientifique

Vie

de produire et d'user de parties, est réservé à la seule création technique (mécanique, électronique, logiciels, etc.), ou s'il est à l'œuvre dans la " pure " activité de notre pensée que serait, par exemple, la philosophie. Cette activité pure n'est-elle pas l'occasion de production et d'assemblage de parties, et les énoncés philosophiques, qui sont des constructions comme l'a montré ailleurs Daniel Parrochia, ne sontils pas le résultat d'un assemblage de parties, ne sontils pas producteurs de patterns que nous pouvons utiliser au mieux pour des finalités particulières ? On peut rechigner à une telle proposition, car ce serait admettre que les philosophies ne sont pas seulement des énoncés dont l'efficacité tient de leur cohérence intrinsèque, mais aussi des objets qui contiennent de I'efficace que l'on peut prélever, transporter et assembler pour fabriquer un autre objet. Et cette vision de l'objet théorique serait valide pour tous les énoncés des sciences humaines. Mais la nature de cette efficacité des parties est-elle la même que celle de l'efficacité dans les logiciels, ou celle de l'efficacité que I'on trouve dans les théories des sciences de la nature ou dans leurs parties?

La production de parties est une action qui entraîne à produire des déchets, ces parties qui ne seront pas utilisées, mais déposées à l'extérieur du système de conception. Ces déchets sont incarnés dans toutes sortes de matière. À côté des déchets de l'industrie par exemple, on doit nécessairement compter avec les déchets de la production philosophique, c'est-à-dire des déchets de la pensée. Les concepteurs de logiciels produisent aussi des déchets. Se pose alors la question de leur gestion. On peut éviter d'en produire, mesurer par avance leur effet sur l'environnement extérieur, ou bien, ne sachant qu'en faire, prévoir de les stocker. La conception de logiciels et l'écoconception $^{5}$ se posent des problèmes analogues, et tous les domaines de l'activité humaine devraient explorer le monde de leurs déchets.

Produire des déchets est une activité humaine et la

${ }^{5}$ La $\approx$ Conception de logiciels * où a été évoquée la production de " déchets dans le cycle de conception d'un programme informatique, et "L'écoconception s ont été le thème de deux des six ateliers du colloque. Les autres thèmes sont :

* Conception centrée utilisateur , " Conception de produits nouveaux $"$, * Génie civil, urbanisme, architecture $: \mathrm{et}$

«Conception d'organisation \%. Les séances plénières ont été réparties en trois thèmes : « Simon

et l'épistémologie de la conception *

* Epistémologie de la conception ,

* Méthodologie de la conception s.

6 Roger Bastide Anthropologie appliquée, éd. originale 1971, 1998 Payot, pp. 172 et sq. trace de la pensée humaine en action, pensée que nous devons explorer comme nous incite à le faire H. Simon. La pensée en action incarnée dans la philosophie produit des " déchets philosophiques ", c'est notre hypothèse, et nous pensons que la pensée en acte produit toujours des déchets. Nous n'allons pas audelà des hypothèses bien argumentées d'Armand Hatchuel et de Benoît Weil. En distinguant, dans leur modèle C-K (Concepts-Knowledge), deux espaces séparés, celui des concepts et celui de la connaissance, ils décrivent la conception comme la co-évolution de ces deux espaces, et généralisent l'algorithme de séparation-évolution décrit par $\mathrm{H}$. Simon. Mais surtout, à notre avis, les deux auteurs montrent que l'aboutissement d'un raisonnement de conception est aussi un double espace de concepts inachevés et de connaissances non utilisées.

Nous devons alors faire l'hypothèse que le processus de conception est agi tant dans l'espace extérieur à l'Homme que de manière isomorphe dans sa pensée. Et si, hardiment, nous relions ce modèle à celui développé dans le colloque par T.M. De Jong, une vision fractale de la conception selon laquelle à tous les niveaux d'observation du processus est joué le pro- cessus, nous obtenons une représentation de ce que décrit $\mathrm{E}$. Morin une polymachine particulière. L'intérêt du modèle C-K n'est pas tant son efficacité que le fait de changer l'une des problématiques posée par Simon en donnant d'emblée la réponse : les mécanismes de la pensée que nous devrions découvrir grâce à l'observation des processus comportementaux des Hommes dans le design sont les processus de la conception, ou d'un certain modèle de ce qu'est concevoir pour un Homme.

Ce n'est pas sans rappeler le principe de récurrence décrit, c'est un exemple, par l'anthropologue Roger Bastide6, qui fait que la nature humaine devient ce que I'on pense qu'elle est, que nous nous transformons selon l'image que nous avons de nous-mêmes. Et ce qui est vrai de l'individu est vrai de la société.

C'est bien de ce principe dont il est question tant dans l'œuvre d'Edgar Morin que dans celle, faut-il le rappeler, du philosophe Gilles Deleuze. La nature humaine est celle que l'Homme construit et s'impose, l'imposant à tout l'environnement, à travers la saisie du monde par l'un de ses modèles. Cela plaide en faveur d'un Homme simple, I'Homme sans qualités de nature qui acquiert ses compétences, ses richesses en produisant et en étant modifié par l'acte de produire et par sa production, en raison de l'isomorphie du processus à tous les niveaux de l'action. L'Homme d'Herbert Simon serait-il un être proche de l'Homme sans qualités de l'écrivain Robert Musil ?

Edgar Morin avait déjà signalé toute la fécondité du processus qui conduit à la création de déchets : ce qui est rejeté par un point de vue, une organisation du monde, devient central dans une autre organisation du monde. À notre connaissance, ces mondes se sont succédés non pas à la suite d'une génération du suivant par le précédent, mais par suite de différents mécanismes de compétition et de sélection. La pensée complexe, paradigme tant $\mathrm{d}^{\prime} \mathrm{E}$. Morin que d'H. Simon, c'est, peut-être, la présence concomitante de tous les modèles possibles assurant la diversité des points de vue. Il y a nécessairement agrégation des modèles dans la pensée humaine et nous devons en être conscients plutôt que d'éliminer ceux qui nous contrarient.

C'est une hypothèse qui pose problème. En effet, I'un des processus les plus fiables que nous ayons pour faire progresser la connaissance est l'élimination des modèles, ou théories, par le processus de falsification que décrit Karl Popper. Or, si nous devions garder tout modèle au nom de la variété des points de vue produits par la pensée humaine, saurions-nous produire de nouveaux modèles et continuer ce processus de transformation de l'Homme que nous venons de décrire? Or, lorsque nous concevons, nous éliminons les produits au moyen de différentes règles, c'est-à-dire nous produisons des déchets. Même la pensée complexe est le résultat d'une production, donc source de déchets, occasion de bâtir un autre modèle. II est donc bon, si notre compréhension d'Edgar Morin est correcte, de produire des déchets, donc d'utiliser tous les outils possibles pour éliminer un modèle, mais il nous faut montrer comment l'on procède, parce qu'ainsi nous donnons à voir des manifestations de notre pensée et, rejoignant Simon, nous réalisons I'un 
des buts des sciences de l'artificiel, comprendre, comme l'être humain, dans l'action.

Nous avons donc tout intérêt à agir avec l'œuvre de Simon comme nous le faisons avec tout autre produit. Nous ne pouvons pas la conserver sans la transformer et tenter de concevoir autre chose à partir d'elle et de ses déchets, car il doit bien y avoir des déchets produits par l'ouvrage du père des sciences de l'artificiel.

${ }^{7}$ ICTT, INSA de Lyon,

Voilà de quoi compléter un programme de jacques.perrin@insa-lyon.fr recherche pour les sciences de l'artificiel appelé de ses ${ }^{8}$ Mécatronique 3 M, UTBM, vœux par Jacques Perrin 7 , l'organisateur avec Joëlle jean-pierre.micaelli@utbm.fr Forest, Jean-Pierre Micaëlli ${ }^{8}$ et Samuel Deniaud ${ }^{9}$ de ce ${ }^{9}$ Mécatronique3M, UTBM, colloque dont nous venons de rendre compte. 